Pacific Journal of Mathematics

AN ESTIMATE FOR DIFFERENTIAL POLYNOMIALS IN
\[ / \partial z_{1}, \cdots, \partial / \partial z n \] 


\section{AN ESTIMATE FOR DIFFERENTIAL POLYNOMIALS}

$$
\text { IN } \frac{\partial}{\partial z_{1}}, \cdots, \frac{\partial}{\partial z_{n}}
$$

François Treves

This article is concerned with polynomials with respect to the CauchyRiemann operators

$$
\frac{\partial}{\partial z_{1}}=\frac{1}{2}\left(\frac{\partial}{\partial x_{1}}+i \frac{\partial}{\partial y_{1}}\right), \cdots, \frac{\partial}{\partial z_{n}}=\frac{1}{2}\left(\frac{\partial}{\partial x_{n}}+i \frac{\partial}{\partial y_{n}}\right) .
$$

We establish an $L^{2}$-estimate, for such polynomials, and derive from it uniqueness in a class of Cauchy problems. The estimate is quite similar to Hörmander's inequalities and, in fact, can be essentially deduced from them. However, its direct proof is very simple and leads to a constant better than the one in Hörmander's inequalities. We have therefore preferred to present it thoroughly.

The last part of the paper studies a class of Cauchy problems and applies the estimate to obtain uniqueness. There the methods are quite standard (see for instance Nirenberg [1]). The nature of the differential operators considered allows us to remove the strict convexity of the domains in which the solutions are studied, and replace it by a weaker condition.

1. The inequality. We consider a polynomial $P(z)$ on $C^{n}$. We set, for $p=\left(p_{1}, \cdots, p_{n}\right) \in N^{n}$ :

$$
P^{(p)}(z)=\left(\frac{\partial}{\partial z_{1}}\right)^{p_{1}} \cdots\left(\frac{\partial}{\partial z_{n}}\right)^{p_{n}} P(z) .
$$

We shall denote by $P\left(D_{z}\right)$ the differential polynomial on $R^{2 n}$ obtained by substituting $\partial / \partial z_{j}=1 / 2\left(\partial / \partial x_{j}+(1 / i)\left(\partial / \partial y_{j}\right)\right)$ for $z_{j}(1 \leqq j \leqq n)$ in $P(z)$.

If $S$ is a subset of $R^{2 n}$, we denote by $\beta_{j}(S)$ the diameter of $S$ in the complex "direction" $z_{j}: \beta_{j}(s)=\sup _{z^{\prime}, z^{\prime \prime} \in S}\left|z_{j}^{\prime}-z_{j}^{\prime \prime}\right|$.

THEOREM 1. Let $\Omega$ be an open set in $R^{2 n}$. For all polynomials $P(z)$ on $C^{n}$, all functions $H(z)$ defined and holomorphic in $\Omega$, all functions $\phi(x, y) \in C_{0}^{\infty}(\Omega)$, all $p=\left(p_{1}, \cdots, p_{n}\right) \in N^{n}$ :

$$
\left\|e^{H(z)} P^{(p)}\left(D_{z}\right) \phi\right\|_{L^{2}} \leqq \beta_{1}^{p_{1}}(\Omega) \cdots \beta_{n}^{p_{n}}(\Omega)\left\|e^{H(z)} P\left(D_{z}\right) \phi\right\|_{L^{2}} .
$$

It is enough to prove the inequality in Theorem 1 for $p_{1}=1$ and $p_{j}=0$ for $j \geqq 2$. We shall denote by $P_{1}(z)$ the corresponding $P^{(p)}(z)$. On the other hand, we set, for $j=1, \cdots, n$ :

Received January 15, 1960. 


$$
\begin{aligned}
& H_{\jmath}(z)=\frac{\partial}{\partial z_{\jmath}} H(z), \\
& A_{\jmath}=\frac{\partial}{\partial z_{\jmath}}-H_{\jmath}(z) .
\end{aligned}
$$

Observe that for all $1 \leqq j, k \leqq n,\left(\partial / \partial z_{j}\right) H_{k}(z)=\left(\partial / \partial z_{k}\right) H_{j}(z)$; it follows from this that the $A_{j}$ 's all commute.

The formal adjoint of $A_{j}$ is $A_{j}^{*}=-\partial / \partial \bar{z}_{j}-\overline{H_{j}(z)}$. Observe first that the $A_{j}^{*}$ 's all commute, since the $A_{j}$ 's do. But also the $A_{j}^{*}$ 's commute with the $A_{k}$ 's, for the $\overline{H_{j}(z)}$ 's are antiholomorphic functions of $\bar{z}$ in $\Omega$.

If $Q(z)$ is a polynomial on $C^{n}$, we denote by $Q(A)$ the differential operator on $R^{2 n}$ obtained by substituting $A_{j}$ for $z_{j}(1 \leqq j \leqq n)$ in $Q(z)$. If $\bar{Q}(z)$ is the polynomial whose coefficients are the complex conjugates of the ones of $Q(z)$, the formal adjoint of the operator $Q(A)$ is $Q^{*}(A)=$ $\bar{Q}\left(A^{*}\right)=\bar{Q}\left(A_{1}^{*}, \cdots, A_{n}^{*}\right)$. It is easy to check that:

$$
\left(P_{j}\right)^{*}(A)=-\left(P^{*}\right)_{j}(A)=-\left[P^{*}(A), \bar{z}_{j}\right] .
$$

Let us denote by (, ) and \|\| the inner product and the norm in $L^{2}\left(R^{2 n}\right)$. We may as well assume that $\beta_{1}(\Omega)=2 d$, with $d=\sup _{z \in \Omega}\left|z_{1}\right|$. If $\phi(x, y)$ has its support in $\Omega$, we can write:

$$
\begin{aligned}
\left(P^{*}(A) \phi, z_{1}\left(P_{1}\right)^{*}(A) \phi\right) & =\left(\phi, P(A)\left[z_{1}\left(P_{1}\right)^{*}(A) \phi\right]\right) \\
& =\left(\bar{z}_{1} \phi,\left(P_{1}\right)^{*}(A) P(A) \phi\right)+\left(\phi,\left(P_{1}\right)^{*}(A) P_{1}(A) \phi\right) \\
& =\left(P_{1}(A)\left(\bar{z}_{1} \phi\right), P(A) \phi\right)+\left\|P_{1}(A) \phi\right\|^{2} \\
& =\left(P_{1}(A) \phi, z_{1} P(A) \phi\right)+\left\|P_{1}(A) \phi\right\|^{2} .
\end{aligned}
$$

Hence:

$$
-\left\|P_{1}(A) \phi\right\|^{2}=\left(P_{1}(A) \phi, z_{1} P(A) \phi\right)+\left(\bar{z}_{1} P^{*}(A) \phi,\left(P^{*}\right)_{1}(A) \phi\right),
$$

by applying (1). We get at once:

( 2 ) $\left\|P_{1}(A) \phi\right\|^{2} \leqq d\left\|P_{1}(A) \phi\right\| \cdot\|P(A) \phi\|+d\left\|P^{*}(A) \phi\right\| \cdot\left\|\left(P^{*}\right)_{1}(A) \phi\right\|$.

But since the $A_{j}$ and the $A_{k}^{*}$ all commute with each other, $P(A)$ and $P^{*}(A)$ commute, and $P_{1}(A)$ and $\left(P_{1}\right)^{*}(A)$ do. Therefore:

$$
\left\|P^{*}(A) \phi\right\|=\|P(A) \phi\|, \quad\left\|\left(P_{1}\right)^{*}(A) \phi\right\|=\left\|P_{1}(A) \phi\right\| .
$$

These relations, together with (2), lead to:

$$
\left\|P_{1}(A) \phi\right\| \leqq(2 d)\|P(A) \phi\| \text {. }
$$

In this inequality (3), let us replace $\phi$ by $e^{H(z)} \phi$; we have

$$
A_{j}\left[e^{H(z)} \phi\right]=e^{H(z)} \frac{\partial \phi}{\partial z_{\jmath}},
$$


and hence:

$$
Q(A)\left[e^{H(z)} \phi\right]=e^{H(z)} Q\left(D_{z}\right) \phi,
$$

for any polynomial $Q(z)$ on $C^{n}$. Thus, we get, from (3):

$$
\left\|e^{H(z)} P_{1}\left(D_{z}\right) \phi\right\| \leqq(2 d)\left\|e^{H(z)} P\left(D_{z}\right) \phi\right\| \text { q.e.d. }
$$

2. Uniqueness in Cauchy problems. We shall denote by $B_{a}(a>0)$ the open ball $|z|<a$ in $C^{n}$.

We say that an open set $\Omega$ in $R^{2 n}$ is admissible at the point $z_{0}$ if $z_{0}$ lies on the boundary of $\Omega$, if the boundary of $\Omega$ is, near $z_{0}$, a piece of a $C^{\infty}$ hypersurface and if the following property holds:

(A) For some $a>0$, there exists a function $F(z)$, holomorphic in the ball $\left|z-z_{0}\right|<a$, vanishing at $z_{0}$ and such that the diameter of the set $U_{b}$ of those points $z \in \Omega$ which satisfy $\left|z-z_{0}\right|<a,-b<\operatorname{Re} F(z)$ converges to 0 when $b>0$ does.

In the sequel, $\Omega$ will be an open set in $R^{2 n}$ admissible at the origin, $a$ will be a positive number such that $(A)$ holds for $z_{0}=0$ and some function $F(z)$ holomorphic in $B_{a}$. Furthermore, we shall assume that the intersection of $B_{a}$ with the boundary of $\Omega$ is a piece $S$ of a hypersurface $C^{\infty}$ (passing by 0 ).

Let us clarify a little the geometric situation. Let us denote by $W$ the piece of the hypersurface $\operatorname{ReF}(z)=0$ contained in $B_{a}$. Since $0 \in W \cap \bar{\Omega} \subset U_{b}$ for every $b>0$, we must have $W \cap \bar{\Omega}=W \cap S=\{0\}$. On the other hand, for any $b>0, U_{b} \cup C \Omega$ is a neighborhood of 0 . For, let $\varepsilon>0$ be small so that $|z|<\varepsilon$ implies $|R e F(z)|<b$. If $z \in B_{\varepsilon}, z \notin U_{b}$ only if $z \notin \Omega$. The interior of $U_{b}$ is never empty. For assume it were and let $z$ belong to $U_{b} ; z$ would have a neighborhood $N$ in which $R e F$ would still be $>-b$ and since $z \in \bar{\Omega}, N$ would intersect $\Omega$; obviously $N \cap \Omega$ is contained in the interior of $U_{b}$.

We consider a polynomial $P(z)$ on $C^{n}$, of degree $m \geq 1$, and a partial differential operator on $R^{2 n}$ with continuous coefficients, $Q$, of order $\leqq \mathrm{m}-1$, satisfying the condition:

$$
\left\|e^{H(z)} Q u\right\|_{L^{2}} \leqq K \sum_{p \neq 0}\left\|e^{H(z)} P^{(p)}\left(D_{z}\right) u\right\|_{L^{2}},
$$

for all $H(z)$ holomorphic in $B_{a}$, all $u(x, y) \in C_{0}^{\infty}$ with support in $B_{a}$.

THEOREM 2. Let $U(x, y)$ be a function defined and $C^{m}$ in $\bar{\Omega}$, with zero Cauchy data on $S$, satisfying:

$$
\left|P\left(D_{z}\right) U\right| \leqq|Q U| \text { in } \bar{\Omega} \text {. }
$$

There exists a neighborhood of 0 in which $U$ vanishes identically. 
We keep our previous notations, for a, $F(z)$, etc.

Let us take a function $\beta(z), C^{\infty}$ in $B_{a}$, with the following properties:

$$
\begin{aligned}
& \beta(z)=1 \text { for } z \in B_{a} \text { and }-2 \varepsilon \leqq \operatorname{Re} F(z) \leqq 0 ; \\
& \beta(z)=0 \text { for } z \in B_{a} \text { and }-3 \varepsilon \leqq \operatorname{Re} F(z),
\end{aligned}
$$

where $\varepsilon>0$ is chosen small enough so that the support of $\beta(z)$ intersects $\Omega$ according to a compact set contained in $B_{a}$. That is possible because of property $(A)$; notice that the diameter of the compact set in question goes to 0 when $\varepsilon \rightarrow 0$.

We define now a function $v(z)$ as being equal to $\beta(z) U$ in $\Omega$ and to 0 elsewhere. Notice the following properties of $v$ :

(i) the support of $v$ is compact (and contained in $B_{a} \cap \bar{\Omega}$ );

(ii) $v(z)$ is $m-1$ times continuously differentiable;

(iii) $P\left(D_{z}\right) v=\beta P\left(D_{z}\right) U+R U \varphi$ in $\Omega, R$ being a partial differential operator with $C^{\infty}$ coefficients.

If one extends the definition of $R U$ by 0 outside $\Omega$, it becomes a continuous function in $B_{a}$ since the order of $R$ is at most $m-1$ and the Cauchy data of $U$ were 0 on $S$. On the other hand, $P\left(D_{z}\right) U$ vanishes also on $S$, because of (2) and of the fact that $Q$ is of order $\leqq m-1$. Hence, continuing $\beta P\left(D_{z}\right) U$ by 0 outside $\Omega$ leads again to a continuous function in $B_{a}$. We see thus that $P\left(D_{z}\right) v$ is a continuous function (in $\left.R^{i n}\right)$. This fact, together with properties (i) and (ii), allows us to extend to $v(z)$ the inequality of Theorem 1 . We see that there exists a constant $A$ such that, for all holomorphic functions $H(z)$ in $B_{a}$,

$$
\sum_{p \neq 0}\left\|e^{H(z)} P^{(p)}\left(D_{z}\right) v\right\|_{L^{2}} \leqq A \delta\left\|e^{H(z)} P\left(D_{z}\right) v\right\|_{L^{2}},
$$

$\delta$ being the diameter of the support of $v$. Remember that $\delta \rightarrow 0$ if $\varepsilon \rightarrow 0$. Since, on $U_{2 \varepsilon}, v=U$, by using inequality (1) and (3), we get:

$$
\begin{gathered}
\int_{U_{2 \varepsilon}} e^{2 R e H}\left(|U|^{2}+|Q U|^{2}\right) d x d y \leqq(2 A K \delta)^{2} \int_{U_{2 \varepsilon}} e^{2 R e H}\left|P\left(D_{z}\right) U\right|^{2} d x d y \\
+(2 A K)^{2} \int_{O U_{2 \varepsilon}} e^{2 R e H}\left|P\left(D_{z}\right) v\right|^{2} d x d y .
\end{gathered}
$$

But since $U_{2 \varepsilon} \subset \Omega$, we have the right to substitute $|Q U|$ for $\left|P\left(D_{z}\right) U\right|$ in the first integral of the right hand side; and if we choose $\varepsilon$ small enough so that $(2 A K \delta)^{2}<1 / 2$, we obtain finally:

$$
\int_{\sigma_{\varepsilon}} e^{2 R e H}|U|^{2} d x d y \leqq M \int_{C_{2 \varepsilon}} e^{2 R e H}\left|P\left(D_{z}\right) v\right|^{2} d x d y \text {, }
$$

$M$ being a constant independent of both $H(z)$ and $\varepsilon$. Observe that the integral on the right hand side is actually performed on $U_{3 \varepsilon} \cap C U_{2 \varepsilon}$. Let us take $H(z)=(t / 2) F(z), t>0$. The nature of the domains of integration leads us to: 


$$
e^{-t \varepsilon} \int_{U_{\varepsilon}}|U|^{2} d x d y \leqq M e^{-2 t \varepsilon} \int_{{ } U_{2 \varepsilon}}\left|P\left(D_{z}\right) v\right|^{2} d x d y
$$

or:

$$
\int_{{ }_{J_{\varepsilon}}}|U|^{2} d x d y \leqq M_{1} e^{-t \varepsilon},
$$

where $M_{1}$ does not depend on $t$; we conclude that $U=0$ in $U_{\varepsilon}$, q.e.d.

We end now by a few remarks about admissible sets.

1. Any open set $\Omega$, strictly convex at a boundary point $z_{0}$ (and bounded near $z_{0}$ by a piece of $C^{\infty}$ hypersurface) is admissible at this point. For simplicity, let us assume that $z_{0}=0$, and let $H$ be an hyperplane passing by 0 , such that $\bar{\Omega}$ intersects $H$ only at the origin and lies entirely on one side of $H$ (at least near 0 ). Let $N$ be the unit vector, orthogonal to $H$, which lies on the side of $H$ containing $\Omega$. If $N_{1}, \cdots, N_{n}$ are the complex components of $N$, we may choose, as holomorphic function $F(z)$, the hermitian product $\bar{N}_{1} z_{1}+\cdots+\bar{N}_{n} z_{n}$.

2. There are open sets, admissible at a boundary point, which are not strictly convex at this point. For instance, consider an open set $\Omega$ whose boundary contains the origin (and is a piece of $C^{\infty}$ hypersurface near it) and whose complement contains the cylinder $\left|z_{1}-\alpha\right|<|\alpha|, \alpha$ being a complex number $\neq 0$. If the diameter of the intersection of $\Omega$ with the cylinder $\left|z_{1}-k \alpha\right|<e^{0} k|\alpha|(k<1, \varepsilon>0)$ tends to 0 when $\varepsilon \rightarrow 0, \Omega$ will be admissible at $z=0$. For then we may take, as holomorphic function $F(z)$, any branch of $-\log \left(1-z_{1} / k \alpha\right)$. If $n=1$, any open set whose complement contains the circle $\left|z_{1}-\alpha\right|<|\alpha|$ (and whose boundary, near 0 , is a piece of $C^{\infty}$ curve passing by 0 ) is admissible at $z_{1}=0$. If $n>1$, one may still construct open sets having the desired properties, which are not strictly convex at $z=0$.

3. Let $F(z)$ be any holomorphic function of $z$ in a neighborhood $U$ of 0 in $C^{n}$, such that $F(0)=0$. Let $U_{+}$be the set of points $z \in U$ such that $\operatorname{Re} F(z)>0$. If $n>1$, the set $U_{+}$cannot be strictly convex at $z=0$.

It $U_{+}$were strictly convex at 0 , there should exist an hyperplane $H$, passing by 0 , intersecting $\bar{U}_{+}$only at this point 0 and such that $U_{+}$ would lie only on one side of $H$. Let $\Omega$ be the other side of $H$, and $U(b)$ be the set of $z \in U$ such that $\operatorname{Re} F(z)>-b, \quad(b>0)$. After maybe shrinking $U$ we may say that the diameter of $U(b) \cap \Omega$ converges to 0 when $b \rightarrow 0$. For assume that this were not true: there would be pairs of points $z_{k}^{\prime}, z_{k}^{\prime \prime}$ in $U(1 / k)$ such that $\left|z_{k}^{\prime}-z_{k}^{\prime \prime}\right| \geqq c>0$ for every $k=1,2, \cdots$. We could assume that $z_{k}^{\prime}$ converges to $z^{\prime}, z_{k}^{\prime \prime}$ to $z^{\prime \prime}$, and 
we should have: $\left|z^{\prime}-z^{\prime \prime}\right| \geqq c, z^{\prime}, z^{\prime \prime} \in \bar{\Omega}$. But also $\operatorname{Re} F\left(z^{\prime}\right)=0, \operatorname{Re} F\left(z^{\prime \prime}\right)=0$, i.e., $z^{\prime}, z^{\prime \prime} \in \bar{U}_{+}$. But that implies $z^{\prime}=z^{\prime \prime}=0$, which is absurd. Hence the open set $\Omega$ is admissible at $z=0$. But if $\Omega$ is admissible at some boundary point, the same must clearly be true for any open half space in $C^{n}$. And this would mean that there is uniqueness in the Cauchy problem for data on an arbitrary hyperplane and for any differential polynomial

$$
P\left(\frac{\partial}{\partial z_{1}}, \cdots, \frac{\partial}{\partial z_{n}}\right)
$$

which is absurd.

\section{REFERENCE}

1. L. Nirenberg, Uniqueness in the Cauchy problem..., Comm. Pure Appl. Math., 10 (1957), 85-105. 


\section{PACIFIC JOURNAL OF MATHEMATICS}

\section{EDITORS}

David GILbarg

Stanford University

Stanford, California

\section{F. H. BRowneLL}

University of Washington

Seattle 5, Washington

\section{A. L. Whiteman}

University of Southern California Los Angeles 7, California

\section{J. PAIGe}

University of California

Los Angeles 24, California

\section{ASSOCIATE EDITORS}
E. F. BECKENBACH
T. M. CHERRY
D. DERRY

\author{
E. HEWITT \\ A. HORN \\ L. NACHBIN
}

\author{
M. OHTSUKA \\ H. L. ROYDEN \\ M. M. SCHIFFER
}

E. SPANIER

E. G. STRAUS

F. WOLF

\section{SUPPORTING INSTITUTIONS}

\author{
UNIVERSITY OF BRITISH COLUMBIA \\ CALIFORNIA INSTITUTE OF TECHNOLOGY \\ UNIVERSITY OF CALIFORNIA \\ MONTANA STATE UNIVERSITY \\ UNIVERSITY OF NEVADA \\ NEW MEXICO STATE UNIVERSITY \\ OREGON STATE COLLEGE \\ UNIVERSITY OF OREGON \\ OSAKA UNIVERSITY \\ UNIVERSITY OF SOUTHERN CALIFORNIA
}

\author{
STANFORD UNIVERSITY \\ UNIVERSITY OF TOKYO \\ UNIVERSITY OF UTAH \\ WASHINGTON STATE COLLEGE \\ UNIVERSITY OF WASHINGTON \\ AMERICAN MATHEMATICAL SOCIETY \\ CALIFORNIA RESEARCH CORPORATION \\ HUGHES AIRCRAFT COMPANY \\ SPACE TECHNOLOGY LABORATORIES \\ NAVAL ORDNANCE TEST STATION
}

\footnotetext{
Mathematical papers intended for publication in the Pacific Journal of Mathematics should be typewritten (double spaced), and the author should keep a complete copy. Manuscripts may be sent to any one of the four editors. All other communications to the editors should be addressed to the managing editor, L. J. Paige at the University of California, Los Angeles 24, California.

50 reprints per author of each article are furnished free of charge; additional copies may be obtained at cost in multiples of 50 .
}

The Pacific Journal of Mathematics is published quarterly, in March, June, September, and December. The price per volume (4 numbers) is $\$ 12.00$; single issues, $\$ 3.50$. Back numbers are available. Special price to individual faculty members of supporting institutions and to individual members of the American Mathematical Society: $\$ 4.00$ per volume; single issues, $\$ 1.25$.

Subscriptions, orders for back numbers, and changes of address should be sent to Pacific Journal of Mathematics, 2120 Oxford Street, Berkeley 4, California.

Printed at Kokusai Bunken Insatsusha (International Academic Printing Co., Ltd.), No. 6, 2-chome, Fujimi-cho, Chiyoda-ku, Tokyo, Japan.

PUBLISHED BY PACIFIC JOURNAL OF MATHEMATICS, A NON-PROFIT CORPORATION

The Supporting Institutions listed above contribute to the cost of publication of this Journal, but they are not owners or publishers and have no responsibility for its content or policies. 


\section{Pacific Journal of Mathematics}

\section{Vol. 10, No. $4 \quad$ December, 1960}

M. Altman, An optimum cubically convergent iterative method of inverting a linear bounded operator in Hilbert space . . . . . . . . . . . . . . . . . . . . . . . . . . 1107

Nesmith Cornett Ankeny, Criterion for rth power residuacity ................. 1115

Julius Rubin Blum and David Lee Hanson, On invariant probability measures I . . . . . 1125

Frank Featherstone Bonsall, Positive operators compact in an auxiliary topology ..... 1131

Billy Joe Boyer, Summability of derived conjugate series . . . . . . . . . . . . . . . . 1139

Delmar L. Boyer, A note on a problem of Fuchs . . . . . . . . . . . . . . . . . 1147

Hans-Joachim Bremermann, The envelopes of holomorphy of tube domains in infinite

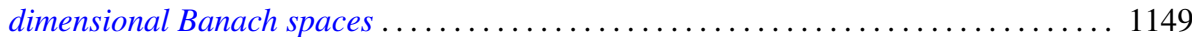

Andrew Michael Bruckner, Minimal superadditive extensions of superadditive

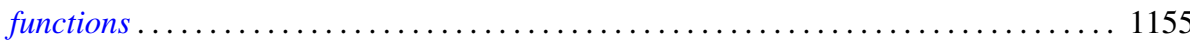

Billy Finney Bryant, On expansive homeomorphisms .................... 1163

Jean W. Butler, On complete and independent sets of operations in finite algebras . . . . . 1169

Lucien Le Cam, An approximation theorem for the Poisson binomial distribution ...... 1181

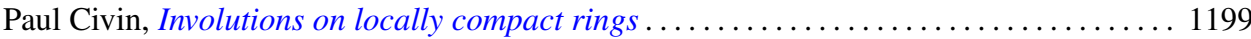

Earl A. Coddington, Normal extensions of formally normal operators . . . . . . . . . 1203

Jacob Feldman, Some classes of equivalent Gaussian processes on an interval ........ 1211

Shaul Foguel, Weak and strong convergence for Markov processes . . . . . . . . . . . 1221

Martin Fox, Some zero sum two-person games with moves in the unit interval ........ 1235

Robert Pertsch Gilbert, Singularities of three-dimensional harmonic functions . . . . . . . 1243

Branko Grünbaum, Partitions of mass-distributions and of convex bodies by

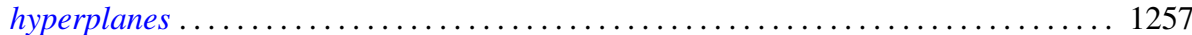

Sidney Morris Harmon, Regular covering surfaces of Riemann surfaces ........... 1263

Edwin Hewitt and Herbert S. Zuckerman, The multiplicative semigroup of integers

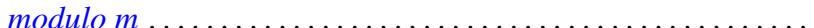

Paul Daniel Hill, Relation of a direct limit group to associated vector groups . ......... 1309

Calvin Virgil Holmes, Commutator groups of monomial groups . .

James Fredrik Jakobsen and W. R. Utz, The non-existence of expansive homeomorphisms

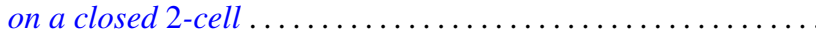

John William Jewett, Multiplication on classes of pseudo-analytic functions . . . . . . . 1323

Helmut Klingen, Analytic automorphisms of bounded symmetric complex domains . . . . 1327

Robert Jacob Koch, Ordered semigroups in partially ordered semigroups . . . . . . . . 1333

Marvin David Marcus and N. A. Khan, On a commutator result of Taussky and

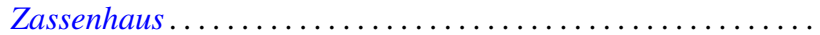

John Glen Marica and Steve Jerome Bryant, Unary algebras......

Edward Peter Merkes and W. T. Scott, On univalence of a continued fraction . . . . . . . 1361

Shu-Teh Chen Moy, Asymptotic properties of derivatives of stationary measures . . . . . 1371

John William Neuberger, Concerning boundary value problems . . . . . . . . . . . 1385

Edward C. Posner, Integral closure of differential rings . . . . . . . . . . . . . . . . . 1393

Marian Reichaw-Reichbach, Some theorems on mappings onto . . . . . . . . . . . . . 1397

Marvin Rosenblum and Harold Widom, Two extremal problems . . . . . . . . . . . . . . . . 1409

Morton Lincoln Slater and Herbert S. Wilf, A class of linear differential-difference

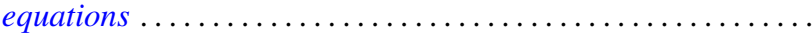

Charles Robson Storey, Jr., The structure of threads . . . . . . . . . . . . . . . . . . 1429

J. François Treves, An estimate for differential polynomials in $\partial / \partial z_{1},, \cdots, \partial / \partial z_{-} n \ldots \ldots 1447$

J. D. Weston, On the representation of operators by convolutions integrals . . . . . . . . 1453

James Victor Whittaker, Normal subgroups of some homeomorphism groups ......... 1469 